\title{
Asymptotics of Nahm sums at roots of unity
}

\author{
Stavros Garoufalidis ${ }^{1} \cdot$ Don Zagier $^{1}$
}

Received: 24 June 2019 / Accepted: 27 February 2020 / Published online: 21 July 2020

(c) The Author(s) 2020

\begin{abstract}
We give a formula for the radial asymptotics to all orders of the special $q$ hypergeometric series known as Nahm sums at complex roots of unity. This result is used in Calegari et al. (Bloch groups, algebraic K-theory, units and Nahm's conjecture. arXiv:1712.04887, 2017) to prove Nahm's conjecture relating the modularity of Nahm sums to the vanishing of a certain invariant in $K$-theory. The power series occurring in our asymptotic formula are identical to the conjectured asymptotics of the Kashaev invariant of a knot once we convert Neumann-Zagier data into Nahm data, suggesting a deep connection between asymptotics of quantum knot invariants and asymptotics of Nahm sums that will be discussed further in a subsequent publication.
\end{abstract}

Keywords Nahm's conjecture $\cdot$ Nahm sums $\cdot q$-Hypergeometric sums $\cdot$ Radial asymptotics $\cdot$ Modular functions $\cdot$ K-theory $\cdot$ Bloch group $\cdot$ Asymptotics $\cdot$ Kashaev invariant

Mathematics Subject Classification Primary - 57N10 - Secondary 57M25

\section{Introduction}

Nahm sums are special $q$-hypergeometric series whose summand involves a quadratic form, a linear form and a constant. They were introduced by Nahm [14] in connection with characters of rational conformal field theories. Nahm formulated a very surprising conjecture, that has elicited a lot of interest, relating the question of their modularity to the vanishing of a certain invariant in algebraic $K$-theory (more specifically, in $K_{3}$ group, or equivalently the Bloch group, of the algebraic numbers). This conjecture is

Stavros Garoufalidis

stavros@mpim-bonn.mpg.de

http://www.math.gatech.edu/stavros

Don Zagier

dbz@mpim-bonn.mpg.de

http://people.mpim-bonn.mpg.de/zagier

1 Max Planck Institute for Mathematics, 53111 Bonn, Germany 
proved in [2] using the asymptotics given in this paper together with the construction of units associated to elements of $K$-theory given there.

The definition of Nahm sums and the question of determining when they are modular were motivated by the famous Rogers-Ramanujan identities, which say that

$$
G(q):=\sum_{n=0}^{\infty} \frac{q^{n^{2}}}{(q)_{n}}=\prod_{\substack{n>0 \\\left(\frac{n}{5}\right)=1}} \frac{1}{1-q^{n}}, \quad H(q):=\sum_{n=0}^{\infty} \frac{q^{n^{2}+n}}{(q)_{n}}=\prod_{\substack{n>0 \\\left(\frac{n}{5}\right)=-1}} \frac{1}{1-q^{n}}
$$

where $(q)_{n}=(1-q) \ldots\left(1-q^{n}\right)$ is the $q$-Pochhammer symbol or quantum $n$-factorial. These identities imply via the Jacobi triple product formula that the two functions $q^{-1 / 60} G(q)$ and $q^{11 / 60} H(q)$ are quotients of unary theta-series by the Dedekind etafunction and hence are modular functions. More generally, Nahm [14] considered the following multi-dimensional generalization:

$$
F_{Q}(q)=F_{A, B, C}(q)=\sum_{n \in \mathbb{Z}_{\geq 0}^{N}} \frac{q^{Q(n)}}{(q)_{n_{1}} \cdots(q)_{n_{N}}} \in \mathbb{Z}\left(\left(q^{\frac{1}{d}}\right)\right)
$$

where $Q: \mathbb{Z}^{N} \rightarrow \mathbb{Q}$ is a quadratic function, i.e., a function of the form

$$
Q(n)=\frac{1}{2} n^{t} A n+B n+C,
$$

where $A=\left(a_{i j}\right)$ is a symmetric positive definite $N \times N$ matrix with rational entries, $B \in \mathbb{Q}^{N}$ a column vector and $C \in \mathbb{Q}$ a scalar and $d$ is any denominator of $Q$ (i.e., any positive integer with $\left.d Q\left(\mathbb{Z}^{N}\right) \subseteq \mathbb{Z}\right)$.

Our aim is to give the asymptotic expansion of $F_{Q}(q)$ as $q$ approaches a root of unity (of order prime to a denominator of $Q$ ) radially.

The constant term of these asymptotic expansions is used in [2] to prove Nahm's modularity conjecture. Very strikingly, our formulas are identical to a collection of power series (one for every complex root of unity) associated to a Neumann-Zagier datum in $[3,4]$ and conjectured to be the asymptotic expansion of the Kashaev invariant at complex roots of unity. The coincidence of the asymptotics of Nahm sums at $q=1$ and the series of [3] was observed several years ago via an explicit map from Neumann-Zagier data to Nahm data, and leads to a deeper connection between quantum invariants of knots defined on the roots of unity (such as the Kashaev invariant) and $q$-series invariants of knots (such as the 3D-index of Dimofte-Gukov-Gaiotto [6]). This connection will be explained in a later publication [10].

Nahm sums appear naturally in cohomological mirror symmetry [12], in the representation theory of quivers [13] and in quantum topology in relation to the stabilization of the coefficients of the colored Jones polynomial [9]. In addition they are building blocks of the 3D-index of an ideally triangulated manifold due to Dimofte-GaiottoGukov [5,6], and appear as holomorphic blocks in the state-integrals of Chern-Simons theory with complex gauge group [1,8]. Further connections between quantum topological invariants and Nahm sums are given in [10]. 


\section{Asymptotic formula for the summand of a Nahm sum}

Recall the Pochhammer symbol $(q x ; q)_{\infty}=\prod_{i \geq 1}\left(1-q^{i} x\right)$, an entire function of $x$ for $q$ a complex number with $|q|<1$. Lemma 2.1 below gives the radial asymptotics of the Pochhammer symbol at roots of unity. To formulate it, recall the $r$ th Bernoulli polynomial $B_{r}(x)$ with generating series $t e^{t x} /\left(e^{t}-1\right)=\sum_{r=0}^{\infty} B_{r}(x) t^{r} / r$ ! , the $r$ th polylogarithm function $\operatorname{Li}_{r}(w)=\sum_{k \geq 1} w^{k} / k^{r}$ for $|w|<1$ and the cyclic quantum dilogarithm function

$$
D_{\zeta}(x)=\prod_{t=1}^{m-1}\left(1-\zeta^{t} x\right)^{t} \in \mathbb{Q}(\zeta)[x]
$$

where $\zeta$ is a primitive $m$ th root of unity. The function that we will actually use is $D_{\zeta}(x)^{1 / m}$ when $|x|<1$, where the $m$ th root is defined by using the principal part of the logarithm of each factor in (2). Below, if $f(\varepsilon)$ is the germ of a smooth function of $\varepsilon$ defined in a neighborhood of 0 in the right half-plane $\Re(\varepsilon)>0$, we write that

$$
f(\varepsilon) \sim \sum_{k=0}^{\infty} a_{k} \varepsilon^{k}
$$

for $\varepsilon \searrow 0$ if $f(\varepsilon)=\sum_{k=0}^{K-1} a_{k} \varepsilon^{k}+\mathrm{O}\left(\varepsilon^{K}\right)$ for every $K>0$ as $\varepsilon$ tends to 0 from the right, or equivalently if $f$ is $C^{\infty}$ from the right at 0 with Taylor coefficients $a_{k}=f^{(k)}(0) / k !$.

Lemma 2.1 Let $w$ be a complex number with $|w|<1, q=\zeta e^{-\varepsilon / m}$ where $\zeta$ is a primitive mth root of unity, and $v$ a complex number such that $v \varepsilon=o(1)$. Set

$$
\begin{aligned}
\log \left(q w e^{-\frac{v \varepsilon}{m}} ; q\right)_{\infty}= & -\frac{1}{m \varepsilon} \operatorname{Li}_{2}(z)-\left(\frac{v}{m}-\frac{1}{2}\right) \log (1-z)-\frac{\varepsilon v^{2}}{2 m} \frac{z}{1-z} \\
& -\frac{1}{m} \log D_{\zeta}(w)-\log (1-w)+\psi_{w, \zeta}(v, \varepsilon)
\end{aligned}
$$

where $z=w^{m}$. Then, $\psi_{w, \zeta}(\nu, \varepsilon)$ has an explicit asymptotic expansion in $\mathbb{C}\left[v \varepsilon^{2 / 3}\right]\left[\left[\varepsilon^{1 / 3}\right]\right] \cap \mathbb{C}[v][[\varepsilon]]$ as $\varepsilon \searrow 0$,

$$
\psi_{w, \zeta}(\nu, \varepsilon) \sim-\sum_{r \geq 2} \sum_{t=1}^{m}\left(B_{r}\left(1-\frac{t+v}{m}\right)-\delta_{r, 2} \frac{v^{2}}{m^{2}}\right) \operatorname{Li}_{2-r}\left(\zeta^{t} w\right) \frac{\varepsilon^{r-1}}{r !}
$$

in which the coefficient of $v^{n}$ is $O\left(\varepsilon^{2 n / 3}\right)$ for every $n \geq 0$.

Fix a symmetric, positive definite $N \times N$ matrix $A$ with rational entries and let $\left(z_{1}, \ldots, z_{N}\right)$ denote the unique solution in $(0,1)^{N}$ of Nahm's equation

$$
1-z_{i}=\prod_{j=1}^{N} z_{j}^{A_{i j}} \quad(i=1, \ldots, N)
$$


We define a real number

$$
\Lambda=-\sum_{j=1}^{N} \mathrm{~L}(z)
$$

where $\mathrm{L}(z)$ is the Rogers dilogarithm function (shifted by a constant to make $\mathrm{L}(1)=0$ ), defined for $0<z<1$ by

$$
\mathrm{L}(z)=\mathrm{Li}_{2}(z)+\frac{1}{2} \log (z) \log (1-z)-\frac{\pi^{2}}{6} .
$$

Let

$$
\widetilde{A}=A+\operatorname{diag}(z /(1-z))
$$

where $\operatorname{diag}(z /(1-z))$ denotes the diagonal matrix with diagonal elements $z_{i} /\left(1-z_{i}\right)$.

Proposition 2.2 Fix $k=\left(k_{1}, \ldots, k_{N}\right) \in(\mathbb{Z} / m \mathbb{Z})^{N}$. Consider natural numbers $n_{i} \in$ $\mathbb{N}$ for $i=1, \ldots, N$ satisfying $n_{i} \equiv k_{i} \bmod m$ for $i=1, \ldots, N$ and write $n_{i}=$ $\frac{1}{\varepsilon} \log \frac{1}{z_{i}}+\frac{1}{\sqrt{\varepsilon}} x_{i}$ where $z=\left(z_{1}, \ldots, z_{N}\right) \in(0,1)^{N}$ is the distinguished solution of Nahm's equation and $q=\zeta e^{-\varepsilon / m}$. Then

$$
\begin{aligned}
\frac{e^{-\varepsilon Q(n) / m}}{\prod_{i=1}^{N}(q)_{n_{i}}}=\left(\frac{\varepsilon}{2 \pi}\right)^{\frac{N}{2}} e^{\frac{\Lambda}{m \varepsilon}} \prod_{i=1}^{N} \theta_{i}^{B_{i}}\left(1-z_{i}\right)^{\frac{1}{2}-\frac{1}{m}} \prod_{i=1}^{N} D_{\zeta}\left(\theta_{i}\right)^{-\frac{1}{m}} \prod_{i=1}^{N} \frac{\theta_{i}^{(A k)_{i}}}{\left(\theta_{i} ; \zeta\right)_{k_{i}}} \\
\times e^{-\frac{1}{m} x^{t} \widetilde{A} x} e^{\frac{1}{m} B^{t} x \varepsilon^{1 / 2}-\frac{1}{m} C \varepsilon} \prod_{i=1}^{N} \exp \left(\psi_{\zeta^{k_{i}} z_{i}^{\frac{1}{m}}, \zeta}\left(x_{i} \varepsilon^{-1 / 2}, \varepsilon\right)\right)
\end{aligned}
$$

where $\theta_{i}=z_{i}^{1 / m_{i}} \in(0,1)$. When $\left|x_{i}\right| \leq \varepsilon^{\lambda-\frac{1}{6}}$ for some $\lambda>0$, then each term of the product of the $\psi$-terms in (10) has an asymptotic expansion in $\mathbb{C}[x]\left[\left[\varepsilon^{1 / 2}\right]\right]$ in which the coefficient of $x^{n}$ is $O\left(\varepsilon^{n}\right)$.

\section{Asymptotic formula for a Nahm sum}

The Nahm sum $F_{Q}(q)$ is a formal Puiseux series with integer coefficients in the variable $q^{\frac{1}{d}}$ (where $d$ is a denominator of $Q$ ) analytic in a finite covering of the punctured open unit disk $0<|q|<1$. It will be convenient to work with the complex-valued function $f_{Q}=f_{A, B, C}$ defined in the upper half-plane by

$$
f_{Q}(\tau)=f_{A, B, C}(\tau)=F_{Q}\left(e^{2 \pi i \tau}\right), \quad \Im(\tau)>0,
$$

with the convention that $\left(e^{2 \pi i \tau}\right)^{\lambda}=e^{2 \pi i \tau \lambda}$ for any $\lambda \in \mathbb{Q}$. Our main Theorem 3.1 concerns the asymptotic expansion of $f_{Q}(\tau)$ at the cusps, i.e., when $\tau$ approaches a 
rational number from above. To formulate our results, we need to introduce some more ingredients, namely the quadratic Gauss sums (which appear in the constant term of the asymptotics), and the formal Gaussian integration which gives the asymptotics to all orders.

Recall the formal Gaussian integration of an analytic function $f(x)$ in $N$ variables $x=\left(x_{1}, \ldots, x_{N}\right) \in \mathbb{C}^{N}$ with values in a power series ring, following the notation of [18]:

$$
\mathbf{I}_{A}[f]=\frac{\int_{\mathbb{R}^{N}} e^{-\frac{1}{2} x^{t} A x} f(x) \mathrm{d} x}{\int_{\mathbb{R}^{N}} e^{-\frac{1}{2} x^{t} A x} \mathrm{~d} x} \sim \sum_{n \geq 0} \frac{1}{2^{n} n !}\left(\Delta_{A}^{n} f\right)(0),
$$

where $\Delta_{A}$ denotes the Laplacian with respect to the quadratic form $x^{t} A x$. In particular, for $1 \times 1$ matrices $A$, the formal Gaussian integration is given explicitly by

$$
\mathbf{I}_{A}\left[\sum_{j=0}^{\infty} c_{j} x^{j}\right] \sim \sum_{\ell=0}^{\infty}(2 \ell-1) ! ! c_{2 \ell} A^{-\ell}
$$

This expression is meaningful if the $c_{n}$ belongs to some power series ring (such as $\left.\mathbb{C}\left[\left[\varepsilon^{1 / 2}\right]\right]\right)$ and the valuation of $c_{n}$ approaches zero as $n$ tends to infinity.

For our asymptotic formulas, we use the formal Gaussian integration (motivated by Proposition 2.2)

$$
I_{Q, \zeta}(k, \varepsilon)=\mathbf{I}_{\frac{1}{m}} \widetilde{A}\left[e^{\frac{1}{m} x^{t} B \varepsilon^{1 / 2}-\frac{1}{m} C \varepsilon} \prod_{i=1}^{N} \psi_{\zeta^{k_{i}} z_{i}^{\frac{1}{m}}, \zeta}\left(x_{i} \varepsilon^{-1 / 2}, \varepsilon\right)\right] \quad\left(k \in(\mathbb{Z} / m \mathbb{Z})^{N}\right),
$$

which is a formal power series in $\varepsilon$ with complex coefficients, where we think of the function inside the argument of $\mathbf{I}$ as an analytic function of $x=\left(x_{1}, \ldots, x_{N}\right) \in \mathbb{C}^{N}$ with values in the power series ring $\mathbb{C}\left[\left[\varepsilon^{1 / 2}\right]\right]$.

We call a positive integer $D$ a strong denominator of $Q$ if the value of $Q(k)$ modulo 1 for $k \in \mathbb{Z}^{N}$ depends only on the residue class of $k$ modulo $D$. (For instance, one can take $D=2 d$ where $d$ is any common denominator of $Q$, i.e., any integer with $d Q\left(\mathbb{Z}^{N}\right) \subseteq \mathbb{Z}$.) The final ingredient is the quadratic Gauss sum

$$
G(Q, \alpha)=\frac{1}{D^{N}} \sum_{k \in(\mathbb{Z} / D \mathbb{Z})^{N}} \mathbf{e}(\bar{\alpha} Q(k)),
$$

where

$$
\mathbf{e}(x)=e^{2 \pi i x},
$$

$\alpha \in \mathbb{Q}$ is prime to $D$ and $\bar{\alpha}$ denotes the reduction of $\alpha$ modulo $D$ and $D$ is any strong denominator of $Q$. The sum on the right is clearly independent of the choice of $D$. 
We now have all the ingredients to formulate our main theorem concerning the radial asymptotics of Nahm sums at roots of unity. Since $f_{A, B, C}(\tau)=\mathbf{e}(C \tau) f_{A, B, 0}(\tau)$, we can restrict to the case $C=0$.

Theorem 3.1 Let $Q(x)=\frac{1}{2} x^{t} A x+B x$ be a quadratic function from $\mathbb{Z}^{N}$ to $\mathbb{Q}$ as above. Fix a rational number $\alpha$ whose denominator $m$ is odd and prime to some denominator of $Q$, and let $\zeta=\mathbf{e}(\alpha)$ denote the corresponding primitive $m$-th root of unity. Let $\theta_{i}=z_{i}^{1 / m} \in(0,1)$, where $\left(z_{1}, \ldots, z_{n}\right)$ as in (6) is the positive real solution of the Nahm equation for $A$. Then the asymptotics of the function $f_{Q}(\tau)$ defined in (11) as $\tau$ tends to $\alpha \in \mathbb{Q}$ is given by

$$
e^{-\frac{\Lambda}{m \varepsilon}} f_{Q}\left(\alpha+\frac{i \varepsilon}{2 \pi m}\right) \sim \frac{\chi^{N}}{m^{N / 2}} c(Q) G(Q, \alpha) S_{Q, \zeta}(\varepsilon) \quad \text { as } \varepsilon \searrow 0,
$$

where $\Lambda \in \mathbb{R}$ is defined by (7), $\chi$ is the 12th root of $\zeta$ defined by $\chi=\mathbf{e}\left(\left(\begin{array}{c}m-1 \\ 2\end{array}\right) \frac{\alpha}{12}\right)$, $c(Q)$ is defined by

$$
c(Q)=\operatorname{det}(\widetilde{A})^{-\frac{1}{2}} \prod_{i=1}^{N} \theta_{i}^{B_{i}}\left(1-z_{i}\right)^{\frac{1}{2}-\frac{1}{m}}
$$

with $\widetilde{A}$ (a positive definite matrix with positive determinant) as in (9), $G(Q, \alpha)$ is the Gauss sum (15) and $S_{Q, \zeta}(\varepsilon)$ is the formal power series in $\varepsilon$ defined by

$$
S_{Q, \zeta}(\varepsilon)=\prod_{i=1}^{N} D_{\zeta}\left(\zeta \theta_{i}\right)^{-\frac{1}{m}} \sum_{k \in(\mathbb{Z} / m \mathbb{Z})^{N}} \zeta^{\overline{Q(k)}} \prod_{i=1}^{N} \frac{\theta_{i}^{(A k)_{i}}}{\left(\zeta \theta_{i} ; \zeta\right)_{k_{i}}} I_{Q, \zeta}(k, \varepsilon)
$$

with $I_{Q, \zeta}(k, \varepsilon)$ as in (14), where $\overline{Q(k)}$ denotes the reduction of $Q(k)$ modulo $m$. Moreover, we have

$$
S_{Q, \zeta}(\varepsilon)^{m} \in F_{m}[[\varepsilon]]
$$

where $F=\mathbb{Q}\left(z_{1}^{\frac{1}{d}}, \ldots, z_{N}^{\frac{1}{d}}\right)$ and $F_{m}$ is the cyclotomic extension $F(\zeta)$ of $F$.

It is the final statement (20) of this theorem, restricted to $\varepsilon=0$, that is used in [2] to prove Nahm's Modularity Conjecture.

Remark 3.2 When $\zeta=1$, the statement and the proof of Theorem 3.1 is valid when $A, B$ and $C$ have real (but not necessarily, rational) entries.

\section{Proof of the asymptotic formulas}

\subsection{Proof of Lemma 2.1}

In this section we give the proof of Lemma 2.1. 
Proof We have

$$
\begin{aligned}
- & \log \left(q w e^{-\frac{\nu \varepsilon}{m}} ; q\right)_{\infty} \\
= & -\sum_{n \geq 1} \log \left(1-q^{n} w e^{-\frac{v \varepsilon}{m}}\right) \\
= & \sum_{k \geq 1} \frac{1}{k} \sum_{n \geq 1}\left(q^{n} w e^{-\frac{\nu \varepsilon}{m}}\right)^{k} \\
& \sim \sum_{k \geq 1} \sum_{t=1}^{m} \frac{\left(\zeta^{t} w\right)^{k}}{k} \frac{e^{-k(v+t) \varepsilon / m}}{1-e^{-k \varepsilon}} \quad \text { sum by } n \equiv t \text { mod } m \\
= & \sum_{k \geq 1} \sum_{t=1}^{m} \frac{\left(\zeta^{t} w\right)^{k}}{k} \sum_{r \geq 0} B_{r}\left(1-\frac{t+v}{m}\right) \frac{(k \varepsilon)^{r-1}}{r !} \quad \text { definition of } B_{r}(x) \\
= & \sum_{r \geq 0} \sum_{t=1}^{m} B_{r}\left(1-\frac{t+v}{m}\right) \operatorname{Li}_{2-r}\left(\zeta^{t} w\right) \frac{\varepsilon^{r-1}}{r !} \quad \text { definition of } \operatorname{Li}_{2-r}(z) .
\end{aligned}
$$

Using the distribution property

$$
\sum_{t=1}^{m} \operatorname{Li}_{r}\left(\zeta^{t} w\right)=m^{1-r} \operatorname{Li}_{r}\left(w^{m}\right)
$$

for the polylogarithm, we see that the $r=0$ and $r=1$ terms are given by

$$
\frac{1}{\varepsilon} \sum_{t=1}^{m} \operatorname{Li}_{2}\left(\zeta^{t} w\right)=\frac{1}{m \varepsilon} \operatorname{Li}_{2}\left(w^{m}\right)
$$

and

$$
\sum_{t=1}^{m}\left(\frac{1}{2}-\frac{v+t}{m}\right) \operatorname{Li}_{1}\left(\zeta^{t} w\right)=\left(\frac{v}{m}-\frac{1}{2}\right) \log \left(1-w^{m}\right)+\frac{1}{m} D_{\zeta}(w)+\log (1-w)
$$

respectively. Finally, we remove a part of the $r=2$ term using

$$
\delta_{r, 2} \frac{\varepsilon}{2} \frac{\nu^{2}}{m^{2}} \sum_{t=1}^{m} \operatorname{Li}_{0}\left(\zeta^{t} w\right)=\frac{\varepsilon \nu^{2}}{2 m} \frac{w^{m}}{1-w^{m}} \quad(r=2)
$$

This proves the asymptotic expansion (5), and concludes the proof of the lemma.

\subsection{Proof of Proposition 2.2}

In this section we give the proof of Proposition 2.2. Let $n_{i}$ be as in the statement of Proposition 2.2 and $q=\zeta e^{-\varepsilon / m}$. It follows that $q^{n_{i}}=w_{i} e^{-v_{i} \varepsilon / m}$ where $w_{i}=\zeta^{k_{i}} \theta_{i}$, 
$\theta_{i}=z_{i}^{\frac{1}{m}}$ and $v_{i}=x_{i} \varepsilon^{-1 / 2}$. Therefore,

$$
\frac{1}{(q)_{n_{i}}}=\frac{\left(q^{n_{i}+1} ; q\right)_{\infty}}{(q ; q)_{\infty}}=\frac{\left(q w_{i} e^{-v_{i} \varepsilon / m} ; q\right)_{\infty}}{(q ; q)_{\infty}} .
$$

For the expansion of the denominator, the modularity of $\eta(z)$ (or alternatively, the Euler-macLaurin formula) implies that when $q=e^{-\varepsilon}$ with $\varepsilon \searrow 0$, we have

$$
\log \left(\frac{1}{(q ; q)_{\infty}}\right)=\frac{\pi^{2}}{6 \varepsilon}-\frac{1}{2} \log \left(\frac{2 \pi}{\varepsilon}\right)-\frac{\varepsilon}{24}+O\left(\varepsilon^{K}\right)
$$

for all $K>0$. For the expansion of the numerator in (21), we use Lemma 2.1 combined with the following identity:

$$
D_{\zeta}\left(\zeta^{k_{i}} \theta_{i}\right)=\frac{\left(\theta_{i} ; \zeta\right)_{k_{i}}^{m} D_{\zeta}\left(\theta_{i}\right)}{\left(1-z_{i}\right)^{k_{i}}}=\frac{\left(\theta_{i} ; \zeta\right)_{k_{i}}^{m} D_{\zeta}\left(\theta_{i}\right)}{\prod_{j=1}^{N} \theta_{j}^{m(A k)_{i}}},
$$

where the first equality follows form the fact that

$$
\frac{D_{\zeta}(\zeta x)}{D_{\zeta}(x)}=\frac{(1-x)^{m}}{1-x^{m}}
$$

and the second equality follows from the fact that $z$ is a solution to Nahm's equation.

Finally, the quadratic form expands as follows:

$$
\begin{aligned}
Q(n)= & Q\left(\frac{1}{\varepsilon} \log \frac{1}{z}+\frac{x}{\sqrt{\varepsilon}}\right) \\
= & \frac{1}{2 \varepsilon^{2}}(\log z)^{t} A \log z-\frac{1}{\varepsilon \sqrt{\varepsilon}} x^{t} A \log z+\frac{1}{2 \varepsilon} x^{t} A x \\
& -\frac{1}{\varepsilon} B^{t} \log z+\frac{1}{\sqrt{\varepsilon}} B^{t} x+C .
\end{aligned}
$$

Using the fact that $z$ satisfies Nahm's equation (6), it follows that

$$
\begin{aligned}
-\frac{\varepsilon}{m} Q(n)= & -\frac{1}{2 m \varepsilon} \log z \cdot \log (1-z)+\frac{1}{m} B^{t} \log z-\frac{\varepsilon}{m} C \\
& -\frac{1}{2 m} x^{t} A x+\frac{1}{m \sqrt{\varepsilon}} x^{t} \log (1-z)+\frac{1}{m} \sqrt{\varepsilon} B^{t} x .
\end{aligned}
$$

The first term in the first line of the above equation converts the dilogarithm by the Rogers dilogarithm. The middle term of the last line of the above equation cancels with one term of (4). The remaining terms combine to conclude (10). This concludes the proof of Eq. (10) in Proposition 2.2.

Fix $\lambda>0$ and let $|x| \leq \varepsilon^{\lambda-\frac{1}{6}}$. Then, we can use the asymptotic expansion (5) of $\psi$ and conclude the claim of the proposition. 


\subsection{Proof of Theorem 3.1}

In this section we give a proof of Theorem 3.1. Our strategy is to split Nahm sums according to congruence classes in which case their summand is a positive real number with a unique peak, and their asymptotics can be studied using several applications of the Poisson summation formula.

Below, $\zeta$ denotes a primitive $m$ th root of unity coprime to $D$, a strong denominator of $Q$. Denote by $a_{n}(q)$ the summand of (1) for $n=\left(n_{1}, \ldots, n_{N}\right) \in \mathbb{Z}_{\geq 0}^{N}$. Clearly, we can split $F_{Q}(q)$ as

$$
F_{Q}(q)=\sum_{k \in(\mathbb{Z} / m \mathbb{Z})^{N}, k^{\prime} \in(\mathbb{Z} / D \mathbb{Z})^{N}} F_{Q}^{\left[k, k^{\prime}\right]}(q),
$$

where

$$
F_{Q}^{\left[k, k^{\prime}\right]}(q)=\sum_{\substack{n \in \mathbb{Z}_{\geq 0}^{N} \\ n \equiv k \bmod m, n \equiv k^{\prime} \bmod D}} a_{n}(q) \quad \text { and } \quad a_{n}(q)=\frac{q^{Q(n)}}{(q)_{n_{1}} \cdots(q)_{n_{N}}}
$$

When $n$ is in a fixed congruence class modulo $m D$, with $n=k \bmod m$ and $n=$ $k^{\prime}$ mod $D$, then $Q(n)$ takes a fixed value modulo $1 / D$ and using the Chinese remainder theorem, we get

$$
\zeta^{Q(n)}=\mathbf{e}\left(\overline{\bar{\alpha}} Q\left(k^{\prime}\right)\right) \zeta^{\overline{Q(k)}}
$$

where $\bar{x}$ and $\overline{\bar{x}}$ denote the reduction of $x$ modulo $m$ and $D$, respectively. that

Set $f_{Q}^{\left[k, k^{\prime}\right]}(\tau)=F_{Q}^{\left[k, k^{\prime}\right]}(\mathbf{e}(\tau))$. When $\tau=\alpha+\frac{i \varepsilon}{2 \pi m}$ (i.e., $\left.q=\zeta e^{-\varepsilon / m}\right)$, it follows

$$
f_{Q}^{\left[k, k^{\prime}\right]}(\tau)=\mathbf{e}\left(\overline{\bar{\alpha}} Q\left(k^{\prime}\right)\right) \zeta^{\overline{Q(k)}} f_{Q, \zeta}^{\left[k, k^{\prime}\right]}(\varepsilon)
$$

where

$$
\begin{gathered}
f_{Q, \zeta}^{\left[k, k^{\prime}\right]}(\varepsilon)=\sum_{\substack{n \in \mathbb{Z}_{\geq 0}^{N} \\
n \equiv k \bmod m, n \equiv k^{\prime} \bmod D}} a_{n}^{+}\left(\zeta e^{-\varepsilon / m}\right) e^{-\varepsilon Q(n) / m} \\
, a_{n}^{+}(q)=\frac{1}{(q)_{n_{1}} \cdots(q)_{n_{N}}}
\end{gathered}
$$

Recall the definition of $f(\varepsilon) \sim g(\varepsilon)$ from (3).

Claim 1 We have

$$
f_{Q, \zeta}^{\left[k, k^{\prime}\right]}(\varepsilon) \sim f_{Q, \zeta}^{[k, 0]}(\varepsilon) .
$$


This follows from an application of the Poisson summation formula discussed below. Assuming this, it follows that

$$
\sum_{k^{\prime} \bmod D} f_{Q, \zeta}^{\left[k, k^{\prime}\right]}(\varepsilon)=f_{Q, \zeta}^{[k]}(\varepsilon)
$$

where

$$
f_{Q, \zeta}^{[k]}(\varepsilon)=\sum_{\substack{n \in \mathbb{Z}_{\geq 0}^{N} \\ n \equiv k \bmod m}} a_{n}^{+}\left(\zeta e^{-\varepsilon / m}\right) e^{-\varepsilon Q(n) / m} .
$$

We now write

$$
f_{Q}(\tau)=F_{Q}\left(e^{2 \pi i \tau}\right)=\sum_{n \in \mathbb{Z}_{\geq 0}^{N}} a_{n}\left(e^{2 \pi i \tau}\right) e^{2 \pi i Q(n) \tau}
$$

Notice that $a_{n}(\mathbf{e}(\tau))$ depends on $\tau$ modulo 1 . Now using a strong denominator $D$ of $Q$ and Combining (24), (26), (28) and (29), we split

$$
f_{Q}\left(\alpha+\frac{i \varepsilon}{2 \pi m}\right) \sim G(Q, \alpha) \sum_{k \in(\mathbb{Z} / m \mathbb{Z})^{N}} \zeta^{\overline{Q(k)}} f_{Q, \zeta}^{[k]}(\varepsilon) .
$$

We now study in detail the asymptotics of $f_{Q, \zeta}^{[k]}(\varepsilon)$ as $\varepsilon \searrow 0$ for fixed $k \in(\mathbb{Z} / m \mathbb{Z})^{N}$. The asymptotic analysis uses Proposition 2.2 (which describes a unimodal property of the summand of $\left.f_{Q, \zeta}^{[k]}(\varepsilon)\right)$ and the Poisson summation formula applied several times described tersely in pp. 53, 54 of [18] and in much more detail in [17].

Let $\frac{1}{\sqrt{\varepsilon}} x_{i}=n_{i}-\frac{1}{\varepsilon} \log \frac{1}{z_{i}}$ and set $x_{i}^{(0)}(\varepsilon)=\left\lfloor-\frac{1}{\varepsilon} \log \frac{1}{z_{i}}\right\rfloor+k_{i}$. So, if $n_{i}=k_{i} \bmod m$, then $x_{i} \in\left(x_{i}^{(0)}(\varepsilon)+m \mathbb{Z}\right) \sqrt{\varepsilon}$. Using Proposition 2.2, and extending $a_{n}^{+}(q)=0$ for $n \in \mathbb{Z}^{N} \backslash \mathbb{Z}_{\geq 0}^{N}$, it follows that

$$
f_{Q, \zeta}^{[k]}(\varepsilon)=\gamma_{Q, \zeta}(\varepsilon) \sum_{x \in\left(x^{(0)}(\varepsilon)+m \mathbb{Z}\right) \sqrt{\varepsilon}} e^{-\frac{1}{m} x^{t} \tilde{A} x} \varphi(x, \varepsilon),
$$

where

$$
\gamma_{Q, \zeta}(\varepsilon)=\left(\frac{\varepsilon}{2 \pi}\right)^{\frac{N}{2}} e^{\frac{\Lambda}{m \varepsilon}} \prod_{i=1}^{N} \theta_{i}^{B_{i}}\left(1-z_{i}\right)^{\frac{1}{2}-\frac{1}{m}} \prod_{i=1}^{N} D_{\zeta}\left(\theta_{i}\right)^{-\frac{1}{m}} \prod_{i=1}^{N} \frac{\theta_{i}^{(A k)_{i}}}{\left(\theta_{i} ; \zeta\right)_{k_{i}}} e^{-C \varepsilon / m}
$$

and

$$
\varphi(x, \varepsilon)=e^{\frac{1}{m} B^{t} x \varepsilon^{1 / 2}} \prod_{i=1}^{N} \exp \left(\psi_{\zeta^{k_{i}} z_{i}^{\frac{1}{m}}, \zeta}\left(x_{i} \varepsilon^{-1 / 2}, \varepsilon\right)\right)
$$


Claim 2 When $\lambda<-1 / 2$, then we have

$$
\sum_{x \in\left(x^{(0)}+m \mathbb{Z}\right) \sqrt{\varepsilon}} e^{-\frac{1}{m} x^{t} \tilde{A} x} \varphi(x, \varepsilon) \sim \sum_{x \in\left(x^{(0)}+m \mathbb{Z}\right) \sqrt{\varepsilon} ;\left|x_{i}\right|<\varepsilon^{\lambda+\frac{1}{2}}} e^{-\frac{1}{m} x^{t} \tilde{A} x} \varphi(x, \varepsilon) .
$$

Claim 3 When $\lambda>-2 / 3$ and $K \in \mathbb{N}$, then we have

$$
e^{-\frac{1}{m} x^{t} \tilde{A} x} \varphi(x, \varepsilon)=e^{-\frac{1}{m} x^{t} \widetilde{A} x}\left(1+\sum_{p=1}^{K} C_{p}(x) \varepsilon^{p / 2}\right)+o\left(\varepsilon^{K(3 \lambda+2)}\right)
$$

where $C_{p}(x)$ are polynomials defined using Lemma 2.1.

Claim 4 If $P$ is a polynomial and when $\lambda<-1 / 2$, then we have

$$
\sum_{x \in\left(x^{(0)}+m \mathbb{Z}\right) \sqrt{\varepsilon} ;\left|x_{i}\right|<\varepsilon^{\lambda+\frac{1}{2}}} P(x) e^{-\frac{1}{2 m} x^{t} \widetilde{A} x} \sim(m \varepsilon)^{-N / 2} \int_{\mathbb{R}^{N}} P(x) e^{-\frac{1}{m} x^{t} \widetilde{A} x} \mathrm{~d} x .
$$

Note that there is a competition of the range of $\lambda$ in Claims 2 and 3 , and it is fortunate that the allowable range is nonempty. All three Claims (31)-(33) follow from an application of the Poisson summation formula explained in detail in pp. 623-625 of [17]. Let us elaborate a bit with some comments on Poisson summation focusing on Claim 4 which states, among other things, that the asymptotics of a sum over a shifted lattice is independent of the shift.

Poisson summation: Suppose that $\phi$ is a $C^{\infty}$-function with more than polynomial decay at infinity, i.e., $|\phi(x)|=o\left(|x|^{K}\right)$ for all $K>0$. Then

$$
\sum_{k \in \mathbb{Z}} \phi((k+\alpha) \varepsilon) \sim \frac{1}{\varepsilon} \widehat{\phi}(0) .
$$

The proof of (34) follows from Poisson summation formula

$$
\sum_{k \in \mathbb{Z}} \phi(k+\alpha)=\sum_{\ell \in \mathbb{Z}} \widehat{\phi}(\ell) \mathbf{e}(\ell \alpha)
$$

which implies that

$$
\sum_{k \in \mathbb{Z}} \phi((k+\alpha) \varepsilon)=\frac{1}{\varepsilon} \sum_{\ell \in \mathbb{Z}} \widehat{\phi}\left(\frac{\ell}{\varepsilon}\right) \mathbf{e}(\ell \alpha) .
$$

Since $\phi$ is $C^{\infty}, \widehat{\phi}(x)=O\left(|x|^{-K}\right)$ for every $K>0$ as $|x| \gg 0$. Consequently, for $\ell \neq 0$, each term of the right-hand side of (35) is exponentially small and so is the sum for all nonzero $\ell$. This proves (34). 
To show Claim 4, we use the Poisson summation formula

$$
\begin{aligned}
& \sum_{x \in\left(x^{(0)}+m \mathbb{Z}\right) \sqrt{\varepsilon}} P(x) e^{-\frac{1}{m} x^{t} \widetilde{A} x} \\
& =\sum_{x \in x^{(0)}+m \mathbb{Z}} P(x \sqrt{\varepsilon}) e^{-\frac{\varepsilon}{2 m} x^{t} \widetilde{A} x} \\
& =\sum_{x \in m \mathbb{Z}} g(x) \mathbf{e}\left(x^{t} x^{(0)}\right) \sim g(0)
\end{aligned}
$$

where $g(x)$ denotes the Fourier transform of $P(x \sqrt{\varepsilon}) e^{-\frac{\varepsilon}{2 m} x^{t} \widetilde{A} x}$. Since

$$
g(0)=\varepsilon^{-N / 2} \int_{\mathbb{R}^{N}} P(x) e^{-\frac{1}{m} x^{t} \widetilde{A} x} \mathrm{~d} x
$$

and since the sum in Claim 4 for $\left|x_{i}\right|>\varepsilon^{\lambda+\frac{1}{2}}$ is $O\left(\varepsilon^{K}\right)$ for all $K>0$, Claim 4 follows. In conclusion, we have shown that

$$
f_{Q, \zeta}^{[k]}(\varepsilon) \sim \frac{\chi^{N}}{m^{N / 2}} c(Q) \prod_{i=1}^{N} D_{\zeta}\left(\zeta \theta_{i}\right)^{-\frac{1}{m}} \prod_{i=1}^{N} \frac{\theta_{i}^{(A k)_{i}}}{\left(\zeta \theta_{i} ; \zeta\right)_{k_{i}}} I_{Q, \zeta}(k, \varepsilon)
$$

where $I_{Q, \zeta}(k, \varepsilon)$ is given by (14). The above proof applies mutanis mutandis to the proof of Claim 1 .

Combining Eqs. (36) and (30) concludes the proof of Theorem 3.1.

\section{A syntactical identity among two collections of formal power series}

In this section we discuss a syntactical identity between two formal power series at each complex root of unity, one introduced in [3] and [4] to describe the conjectural asymptotics of the Kashaev invariant near 1 and near general roots of unity, respectively, and the other being the radial asymptotics of Nahm sums as $q$ tends to a root of unity $\zeta$, as given in [18] for $\zeta=1$ and in the present paper for general $\zeta$. We observed by chance that the asymptotic series found in [18] and in [3] agreed to all orders. This then turned out to be true for all $\zeta$, giving a surprising connection between radial asymptotics of $q$-series and asymptotics of quantum invariants defined at roots of unity that was highlighted in [7] and further discussed in [10]. Formally, this connection can be expressed by the commutativity, for all roots of unity $\zeta$, of the following diagram:

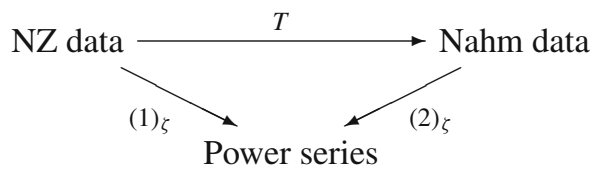

whose ingredients we now explain.

Briefly, Neumann-Zagier (in short, NZ) data are obtained from an ideal triangulation of a cusped hyperbolic 3-manifold $M$ triangulated with $N$ tetrahedra with shapes 
$z=\left(z_{1}, \ldots, z_{N}\right) \in \mathbb{C} \backslash\{0,1\}[15,16]$. The shapes satisfy the NZ equations, which have the form

$$
z^{\mathbf{A}} z^{\prime \prime \mathbf{B}}=e^{-\pi i \eta}
$$

where for a complex number $w$, we define $w^{\prime}=1 /(1-w), w^{\prime \prime}=1-1 / w$ and (A B) is the upper half of a symplectic matrix (i.e., it has full rank and $\mathbf{A B} \mathbf{B}^{t}$ is symmetric) and $\eta \in \mathbb{Z}^{N}$. A solution of (38) in $\mathbb{C} \backslash\{0,1, \infty\}$ gives rise to a PSL(2, $\mathbb{C}$ )-representation of the fundamental group of $M$ and describes the complete hyperbolic structure of $M$ when the solution is in the upper half-plane (i.e., $\Im\left(z_{i}\right)>0$ for all $i$ ). The NZ equations are written for each edge of the triangulation, and for a choice of (meridianlongitude) peripheral curves of each boundary component of $M$. When $M$ has a single torus boundary component, equipped with a meridian and longitude, the matrices $\mathbf{A}$ and $\mathbf{B}$ discussed in [3] we obtained by eliminating the shape $z^{\prime}$ (using the fact that $z z^{\prime} z^{\prime \prime}=-1$ ) giving rise to the vector $\eta$ in (38), and by removing one the edge equations and replacing it by a meridian gluing equation. In addition, a flattening $f \in \mathbb{Z}^{N}$ was introduced and used in [3].

The map $T$ that appears in (37) converts the NZ equation to a Nahm equation. Assuming that $\mathbf{B}$ is nonsingular, we can formally convert (38) in the following form

$$
1-z_{i}=\mathbf{e}\left(B . e_{i}\right) \prod_{j=1}^{N} z_{j}^{A_{i j}} \quad(i=1, \ldots, N)
$$

where $e_{i}$ is the $i$ th coordinate vector, $\mathbf{e}(x)$ is as in (16) and

$$
A=I-\mathbf{B}^{-1} \mathbf{A}, \quad B=\frac{1}{2}\left(-\mathbf{B}^{-1} \eta+(1, \ldots, 1)^{t}\right)
$$

Since (A B) is the upper half of a symplectic matrix, it follows that $A$ is symmetric. This motivates the map $T$ from NZ data to Nahm data

$$
T(\mathbf{A}, \mathbf{B}, \eta, f) \mapsto(A, B, C)
$$

where $C=f$. The transformed equation (39) is the Nahm equation of a twisted Nahm sum $F^{*}$ defined by

$$
F_{A, B, C}^{*}(q)=\sum_{n \in \mathbb{Z}_{\geq 0}^{N}} \mathbf{e}(B . n) \frac{q^{\frac{1}{2} n^{t} A n+B . n+C}}{(q)_{n_{1}} \cdots(q)_{n_{N}}} \in \mathbb{C}\left(\left(q^{\frac{1}{d}}\right)\right),
$$

where $A=\left(a_{i j}\right)$ is a symmetric positive definite $N \times N$ matrix with rational entries, $B \in \mathbb{Q}^{N}$ are column vectors and $C \in \mathbb{Q}$ a scalar.

Now fix a primitive root of unity $\zeta$. The arrow $(1)_{\zeta}$ in (37) is a power series defined in [4] (under the hypothesis that $\mathcal{H}=-\mathbf{B}^{-1} \mathbf{A}+\operatorname{diagonal}\left(z^{\prime}\right)$ is invertible), and the arrow $(2)_{\zeta}$ is the formula of Theorem 3.1 applied formally to the twisted Nahm sum 
$F_{A, B, C}^{*}(q)$ as $q \rightarrow \zeta$. The reason for the commutativity of the diagram (37) is that in $[3,4]$, the formal power series (4) appears due to asymptotic expansion of Faddeev's quantum dilogarithm. The latter is a ratio of two infinite quantum factorials, one in the variable $q=\mathbf{e}(\tau)$ and the other in the variable $\tilde{q}=\mathbf{e}(-1 / \tau)$. Ignoring one of the infinite quantum factorials produces identical power series after formal Gaussian integration.

\section{Coefficient versus radial asymptotics of Nahm sums}

In this section we discuss a relation between the coefficient and the radial asymptotics of an analytic function in the complex unit disk under some fairly weak analytic assumptions which (for instance) are satisfied for the Nahm sums (1).

Consider a function

$$
G(q)=\sum_{n=0}^{\infty} c(n) q^{n}
$$

analytic function in the open complex unit disk $|q|<1$ and with an asymptotic expansion at $q=1$

$$
G\left(e^{-z}\right) \sim e^{C^{2} /(4 z)} \sum_{\alpha}^{\infty} A_{\alpha} z^{\alpha}
$$

as $z \rightarrow 0$ with $\Re(z)>0$ where $C$ is a positive real number and $\alpha$ is a sequence of real numbers tending to infinity. In the case of a Nahm sum, $\alpha \in \mathbb{N}$, and in most applications, $\alpha$ lies in a fixed number of arithmetic progressions of the form $\alpha_{0}+\frac{1}{d} \mathbb{N}$ for $\alpha_{0} \in \mathbb{Q}$ and $d \in \mathbb{N}$. Assume further that for every $N>0$, there exists $\theta_{N}>0$ such that $\theta_{N}=o(N)$ and $\left|G\left(e^{-h+i \theta}\right)\right|<h^{N} e^{C^{2} /(4 h)}$ for $h>0$ (and small) and $|\theta|>\left|\theta_{N}\right|$.

Theorem 6.1 Under the above assumptions, we have

$$
c(n) \sim \frac{1}{2} \sqrt{\frac{C}{2 \pi}} \frac{1}{n^{3 / 4}} e^{C \sqrt{n}} \sum_{\ell \geq 0, \alpha} \frac{(-1)^{\ell}}{C^{\ell-\alpha}}(2 \ell-1) ! !\left(\begin{array}{c}
\alpha+\ell-\frac{1}{2} \\
2 \ell
\end{array}\right) \frac{1}{n^{\frac{\ell}{2}+\frac{\alpha}{2}}} A_{\alpha} .
$$

Note that this implies that the asymptotics of the Fourier coefficients $c(n)$ determine the radial asymptotics $G\left(e^{-h}\right)$ and vice-versa.

Proof The Cauchy residue theorem and the change of variables $q=e^{-z}$ for $z=$ $h+\pi i \theta$ for $\theta \in[-1,1]$ and $h>0$ fixed implies that

$$
c(n)=\frac{1}{2 \pi i} \int_{h-\pi i}^{h+\pi i} e^{n z} G\left(e^{-z}\right) \mathrm{d} z
$$


Using the change of variables $z=C u /(2 \sqrt{n})$ it follows that

$$
c(n) \sim \sum_{\alpha} A_{\alpha} K(\alpha, n)
$$

where the accuracy of the approximation will depend on the accuracy and uniformity of (44) and

$$
\begin{aligned}
K(\alpha, n) & =\frac{1}{2 \pi i} \int_{h-\pi i}^{h+\pi i} \exp \left(\frac{C^{2}}{4 z}+n z\right) z^{\alpha} \mathrm{d} z \\
& =\frac{1}{2 \pi i}\left(\frac{C}{2 \sqrt{n}}\right)^{\alpha+1} \int \exp \left(\frac{C \sqrt{n}}{2}\left(u+\frac{1}{u}\right)\right) u^{\alpha} \mathrm{d} u .
\end{aligned}
$$

The function $K(\alpha, n)$ can be written in closed form in terms of the modified Bessel function as follows: $K(\alpha, n)=K_{\alpha+1}(-C \sqrt{n})$, and the latter has a well-known asymptotic expansion but since the direct calculation of the asymptotic expansion is not difficult, we give it completely here. Make the substitution

$$
u+\frac{1}{u}=2-x^{2}, \quad u=\left(\sqrt{1-x^{2} / 4}+i x / 2\right)^{2}
$$

to make the exponential in (46) a pure Gaussian. Then using the standard binomial coefficient identity

$$
\frac{\mathrm{d}}{\mathrm{d} x}\left(\frac{u^{k}}{k}\right)=\sum_{j=0}^{\infty}(-1)^{j}\left(\begin{array}{c}
k+\frac{j+1}{2} \\
j
\end{array}\right) x^{j}
$$

with $k=\alpha+1$ together with the standard Gaussian integral (13) for $j=2 \ell$ even, we get that

$$
K(\alpha, n) \sim \frac{C^{\alpha+\frac{1}{2}}}{2^{\alpha+\frac{3}{2}} \sqrt{\pi}} e^{C \sqrt{n}} \sum_{\ell=0}^{\infty} \frac{(-1)^{\ell}}{C^{\ell}}(2 \ell-1) ! !\left(\begin{array}{c}
\alpha+\ell-\frac{1}{2} \\
2 \ell
\end{array}\right) n^{-\frac{3}{4}-\frac{\ell}{2}-\frac{\alpha}{2}} .
$$

This completes the proof.

\section{Modular Nahm sums}

In this section we give an application of the asymptotic Theorem 3.1 to the case when the Nahm sum $F_{A, B, C}(q)$ is modular.

Let $X_{A}=\left(X_{A, 1}, \ldots, X_{A, N}\right) \in(0,1)^{N}$ denote the distinguished solution of the Nahm equation $1-X=X^{A}$ and let $\xi_{A} \in B(\mathbb{C})$ denote the corresponding element of the Bloch group and set

$$
C_{0}(A)=-\mathrm{L}\left(\xi_{A}\right) /(2 \pi)^{2}
$$


where $\mathrm{L}$ is our normalization of the Rogers dilogarithm given in (8).

We first make some general remarks about modular functions and their asymptotic properties near rational points. First, by modular function we will always mean a function invariant under a subgroup of finite index of $\operatorname{SL}(2, \mathbb{Z})$. (We do not have to assume that this subgroup is a congruence subgroup, i.e., one containing the principle congruence subgroup $\Gamma(M)$ for some $M \in \mathbb{N}$, although in the case of Nahm sums, which always have an expansion in rational powers of $q$ with integral coefficients, a well-known conjecture implies that if they are modular at all then they are in fact modular with respect to a congruence subgroup.) For any such function $g(\tau)$ and any $P \in \mathbf{P}^{1}(\mathbb{Q})=\mathbb{Q} \cup\{\infty\}$, we define the valuation $v_{P}(g) \in \mathbb{Q}$ of $g$ at $P$ as the smallest exponent of $q=\mathbf{e}(\tau)$ in the Fourier expansion of $(g \circ \gamma)(\tau)$, where $\gamma \in \operatorname{SL}(2, \mathbb{Z})$ is any element such that $\gamma(\infty)=P$. This definition is easily seen to be independent of the choice of $\gamma$.

Recall $f_{Q}(\tau)$ from $(11)$, and let $F_{Q}$ denote $F_{A, B, C}$.

Proposition 7.1 If $f_{Q}(\tau)$ is modular, then for every $P \in \mathbf{P}^{1}(\mathbb{Q})$ we have

$$
v_{P}\left(F_{Q}\right) \geq C_{0}(A)
$$

with equality when $P=0$.

Proof Let $f=f_{Q}, P=a / c$ for $(a, c)=1, c>0$ and $\gamma=\left(\begin{array}{ll}a & b \\ c & d\end{array}\right) \in \operatorname{SL}(2, \mathbb{Z})$. Take $\epsilon>0$ and set $\tau=\left(i \epsilon^{-1}-d\right) / c$ in the upper half-plane $\left(\tau \rightarrow \infty\right.$ as $\left.\epsilon \rightarrow 0^{+}\right)$. Then,

$$
\gamma(\tau)=\frac{a \tau+b}{c \tau+d}=\frac{a}{c}+\frac{i \epsilon}{c}
$$

combined with $q=\mathbf{e}(\tau)=\mathbf{e}\left(i v_{P}(f) /(c \epsilon)\right) \mathbf{e}\left(-d v_{P}(f) / c\right)$ implies that

$$
(f \circ \gamma)(\tau)=f\left(\frac{a+i \epsilon}{c}\right)=C^{\prime} q^{v_{P}(f)}\left(1+O\left(q^{1 / D}\right)\right) \sim C^{\prime} \mathbf{e}\left(i v_{P}(f) /(c \epsilon)\right)
$$

for some $C^{\prime} \neq 0$ and some $D \in \mathbb{Q}_{+}$. On the other hand, Theorem 3.1 with $\zeta=\mathbf{e}(P)$ and $n=c$ implies that

$$
f\left(\frac{a+i \epsilon}{c}\right) \sim C^{\prime \prime} \mathbf{e}\left(i C_{0}(A) /(c \epsilon)\right)
$$

where $C^{\prime \prime}$ is a constant, possibly zero. A comparison between (52) and (53) implies inequality (51). When $P=0$, i.e., $\zeta=1$, Theorem 3.1 asserts that $C^{\prime \prime} \neq 0$. In that case, (52) and (53) imply equality in (51).

As a special case of the proposition, for $P=\infty$ it follows that

$$
v_{\infty}\left(f_{Q}\right)=\min _{n \in \mathbb{Z}_{\geq 0}^{N}}(Q(n)) \geq C_{0}(A)
$$


whenever $f_{Q}$ is modular. If in addition $\frac{1}{2} n^{t} A n+n^{t} B \geq 0$ for all $n \in \mathbb{Z}_{\geq 0}^{N}$ (as is the case for all the modular triples $(A, B, C)$ of rank 1, 2 or 3 listed in [18] and [17]), then $v_{\infty}\left(f_{A, B, C}\right)=C$ and we deduce that $C \geq C_{0}(A)$. Moreover, in all cases observed, the equality $C=C_{0}(A)$ holds if and only if the vector $B$ is zero, and this value occurs whenever the matrix $A$ is integral and even. (The converse to this last statement, however, is not true; for instance, the Nahm sum $f_{A, 0, C_{0}(A)}$ is modular also for $A=\left(\begin{array}{ll}4 / 3 & 2 / 3 \\ 2 / 3 & 4 / 3\end{array}\right)$ or its inverse $A=\left(\begin{array}{cc}1 & -1 / 2 \\ -1 / 2 & 1\end{array}\right)$, as well as for several nonintegral $3 \times 3$ matrices $A$.)

Acknowledgements Open access funding provided by Projekt DEAL.

Open Access This article is licensed under a Creative Commons Attribution 4.0 International License, which permits use, sharing, adaptation, distribution and reproduction in any medium or format, as long as you give appropriate credit to the original author(s) and the source, provide a link to the Creative Commons licence, and indicate if changes were made. The images or other third party material in this article are included in the article's Creative Commons licence, unless indicated otherwise in a credit line to the material. If material is not included in the article's Creative Commons licence and your intended use is not permitted by statutory regulation or exceeds the permitted use, you will need to obtain permission directly from the copyright holder. To view a copy of this licence, visit http://creativecommons.org/licenses/by/4.0/.

\section{Appendix A. Application: proof of the Kashaev-Mangazeev-Stroganov identity}

The current paper is needed crucially in [2], where the asymptotic properties of Nahm sums at roots of unity are used to prove Nahm's conjecture about their modularity. A further essential ingredient in [2] was the following finite version of the 5-term relation for the cyclic quantum dilogarithm due to Kashaev, Mangazeev and Stroganov:

Proposition 8.1 [11, Eq. C.7] Let $X, Y$ and $Z$ be three complex numbers satisfying $Z=\frac{1-X}{1-Y}$ and $\zeta$ a primitive $m$ th root of unity. Then

$$
\frac{D_{\zeta}(1) D_{\zeta}(y \zeta / x) D_{\zeta}(x / y z)}{D_{\zeta}(1 / x) D_{\zeta}(y \zeta) D_{\zeta}(\zeta / z)}=(\zeta y)^{m(1-m) / 2} f(x, y \mid z)^{m},
$$

where $x, y$ and $z$ are $m$ th roots of $X, Y$ and $Z$ and

$$
f(x, y \mid z)=\sum_{k \bmod m} \frac{(\zeta y ; \zeta)_{k}}{(\zeta x ; \zeta)_{k}} z^{k}
$$

An independent proof of the above identity was given in unpublished work of Gangl and Kontsevich. In this appendix we give a simple proof of this identity as an application of the asymptotic formula in Lemma 2.1, or rather of its weakening (take $w=x, v=0$ and retain only the leading terms)

$$
\left(x ; \zeta e^{-\varepsilon / m}\right)_{\infty}^{-m} \sim \frac{D_{\zeta}(x)}{\left(1-x^{m}\right)^{m / 2}} e^{\operatorname{Li}_{2}\left(x^{m}\right) / \varepsilon} \quad\left(x \in \mathbb{C}, x^{m} \notin[1, \infty), \varepsilon \searrow 0\right) .
$$


in combination with a famous identity of Ramanujan. The same method could presumably be used to prove many other identities.

Note that the right-hand side of (56) is well-defined because the relation $Z(1-Y)=$ $1-X$ implies that the summand is $m$-periodic. Furthermore, both sides of (56) are rational functions on the curve $z^{m}\left(1-y^{m}\right)=1-x^{m}$, so it suffices to prove them in an open set of that curve. With this in mind, let $X, Y, Z$ be as in the proposition above but also satisfying that $X, Y \notin \mathbb{R}$ and $|X / Y|<|Z|<1$. Set $x=X^{1 / m}, y=Y^{1 / m}$, and $z=Z^{1 / m}$, and choose $q=\zeta e^{-\varepsilon / m}$ with $\varepsilon>0$ small. The Ramanujan ${ }_{1} \Psi_{1}$ summation formula says that

$$
\begin{aligned}
{ }_{1} \Psi_{1}(x, y, z ; q) \\
\quad:=\sum_{k=-\infty}^{\infty} \frac{(q y ; q)_{k}}{(q x ; q)_{k}} z^{k} \\
\quad=\frac{(q ; q)_{\infty}(q y z ; q)_{\infty}(1 / y z ; q)_{\infty}(x / y ; q)_{\infty}}{(q x ; q)_{\infty}(1 / y ; q)_{\infty}(z ; q)_{\infty}(x / y z ; q)_{\infty}},
\end{aligned}
$$

where $(x ; q)_{k}=\left(q^{k} x ; q\right)_{|k|}^{-1}$ for $k<0$ and where the series converges because of the conditions placed on $X, Y$ and $Z$. Denote by $A_{k}$ the $k$ th summand in the series. Then for fixed $k$ we have

$$
\frac{A_{k+m}}{A_{k}}=\prod_{j=1}^{m}\left(\frac{1-q^{k+j} y}{1-q^{k+j} x} z\right)=\frac{1-Y}{1-X} Z+\mathrm{O}(\varepsilon)=1+\mathrm{O}(\varepsilon) \quad(\varepsilon \searrow 0),
$$

so $A_{k}$ is periodic up to finite order in $\varepsilon$. This implies that the left-hand side of (58) is the sum of $m$ terms each of the form $\sum_{n \in \mathbb{Z}} \phi(n)$ where $\phi(x)$ is an approximate Gaussian centered at $x=0$. If we assume only $k=\mathrm{o}(1 / \varepsilon)$ rather than $k=O(1)$, then we have instead:

$$
\frac{A_{k+m}}{A_{k}}=\frac{1-e^{-k \varepsilon} Y}{1-e^{-k \varepsilon} X} Z+\mathrm{O}(\varepsilon)=1+\left(\frac{Y}{1-Y}-\frac{X}{1-X}\right) k \varepsilon+\mathrm{O}\left(\varepsilon+k^{2} \varepsilon^{2}\right)
$$

It follows that

$$
A_{k+n m}=\frac{(\zeta y ; \zeta)_{k}}{(\zeta x ; \zeta)_{k}} z^{k} \cdot \exp \left(\frac{X-Y}{(1-X)(1-Y)} \frac{n^{2} m}{2} \varepsilon+\mathrm{O}\left(n \varepsilon+n^{3} \varepsilon^{2}\right)\right)
$$

for $k$ fixed and $n=\mathrm{o}(1 / \varepsilon)$. Our assumptions on $(X, Y, Z)$ imply that $\Re\left(\frac{Y-X}{(1-X)(1-Y)}\right)<$ 0 , so the right-hand side behaves like a Gaussian. We deduce that

$$
{ }_{1} \Psi_{1}\left(x, y, z ; \zeta e^{-\varepsilon / m}\right) \sim \sqrt{\frac{2 \pi}{\varepsilon}} \sqrt{\frac{(1-Y)(1-X)}{(X-Y)}} f(x, y \mid z) \quad(\varepsilon \searrow 0),
$$


where $f(x, y \mid z)$ as in Eq. (56). On the other hand, by the transformation formula of the Dedekind eta-function the factor $(q ; q)_{\infty}$ in $(58)$ satisfies the asymptotic formula

$$
(q ; q)_{\infty}=(\mu+\mathrm{O}(\varepsilon)) \sqrt{\frac{2 \pi}{\varepsilon}} e^{-\pi^{2} / 6 m \varepsilon}
$$

for some $(24 m)$ th root of unity $\mu$, and inserting this and the asymptotic formula from (57) into the product in Eq. (58) we find the alternative asymptotic formula

$$
{ }_{1} \Psi_{1}\left(x, y, z ; \zeta e^{-\varepsilon / m}\right)^{m} \sim \sqrt{\frac{2 \pi}{\varepsilon}} C^{m / 2} D e^{B / \varepsilon} \quad(\varepsilon \searrow 0),
$$

where

$$
\begin{aligned}
B= & -\mathrm{Li}_{2}(X / Y Z)+\mathrm{Li}_{2}(Y Z)+\mathrm{Li}_{2}(1 / Y Z)+\mathrm{Li}_{2}(X / Y)+\mathrm{Li}_{2}(1) \\
& -\mathrm{Li}_{2}(X)-\mathrm{Li}_{2}(1 / Y)-\mathrm{Li}_{2}(Z), \\
C= & \frac{(1-Y Z)(1-1 / Y Z)(1-X / Y)}{(1-X)(1-1 / Y)(1-Z)(1-X / Y Z)},
\end{aligned}
$$

and

$$
D=\mu_{24} \frac{D_{\zeta}(\zeta x) D_{\zeta}(1 / y) D_{\zeta}(z) D_{\zeta}(x / y z)}{D_{\zeta}(\zeta y z) D_{\zeta}(1 / y z) D_{\zeta}(x / y)}
$$

where $\mu_{24}=\zeta^{m}$ is a 24 th root of unity. The quantity $B$ vanishes by the standard functional equations of the dilogarithm. Using further the identities

$$
D_{\zeta}(\zeta x)=D_{\zeta}(x) \frac{(1-x)^{m}}{1-x^{m}}, \quad D_{\zeta}(1 / x) D_{\zeta}(x)=\mu_{6} x^{-\frac{m(m-1)}{2}} \frac{\left(1-x^{m}\right)^{m}}{(1-x)^{m}}
$$

(where $\mu_{6}$ is a sixth root of unity) and comparing the asymptotic equations (59) and (60), we get (55) as desired.

\section{References}

1. Beem, C., Dimofte, T., Pasquetti, S.: Holomorphic blocks in three dimensions. J. High Energy Phys. 2014(12), 177 (2014)

2. Calegari, F., Garoufalidis, S., Zagier, D.: Bloch groups, algebraic K-theory, units and Nahm's Conjecture, Preprint 2017. arXiv:1712.04887

3. Dimofte, T., Garoufalidis, S.: The quantum content of the gluing equations. Geom. Topol. 17(3), $1253-1315$ (2013)

4. Dimofte, T., Garoufalidis, S.: Quantum modularity and complex Chern-Simons theory. Commun. Number Theory Phys. 12(1), 1-52 (2018)

5. Dimofte, T., Gaiotto, D., Gukov, S.: 3-Manifolds and 3d indices. Adv. Theor. Math. Phys. 17(5), 975-1076 (2013)

6. Dimofte, T., Gaiotto, D., Gukov, S.: Gauge theories labelled by three-manifolds. Commun. Math. Phys. 325(2), 367-419 (2014)

7. Garoufalidis, Stavros: Quantum knot invariants. Res. Math. Sci. 5(1), Paper No. 11, 17 (2018) 
8. Garoufalidis, S., Kashaev, R.: From state integrals to $q$-series. Math. Res. Lett. 24(3), 781-801 (2017)

9. Garoufalidis, S., Lê, T.T.Q.: Nahm sums, stability and the colored Jones polynomial. Res. Math. Sci. 2, 1 (2015)

10. Garoufalidis, S., Zagier, D.: Knots, quantum modularity, and perturbative series, In preparation

11. Kashaev, R., Mangazeev, V., Stroganov, Y.: Star-square and tetrahedron equations in the BaxterBazhanov model. Int. J. Mod. Phys. A 8(8), 1399-1409 (1993)

12. Kontsevich, M., Soibelman, Y.: Cohomological Hall algebra, exponential Hodge structures and motivic Donaldson-Thomas invariants. Commun. Number Theory Phys. 5(2), 231-352 (2011)

13. Kucharski, P., Reineke, M., Stošić, M., Sulkowski, P.: BPS states, knots, and quivers. Phys. Rev. D 96(12), 121902(R), 6 (2017)

14. Nahm, W.: Conformal Field Theory and Torsion Elements of the Bloch Group, Frontiers in Number Theory, Physics, and Geometry. II, pp. 67-132. Springer, Berlin (2007)

15. Neumann, W.D., Zagier, D.: Volumes of hyperbolic three-manifolds. Topology 24(3), 307-332 (1985)

16. Thurston, W.: The geometry and topology of 3-manifolds. Universitext, Springer, Berlin, 1977, Lecture notes, Princeton

17. Vlasenko, M., Zwegers, S.: Nahm's conjecture: asymptotic computations and counterexamples. Commun. Number Theory Phys. 5(3), 617-642 (2011)

18. Zagier, D.: The Dilogarithm Function, Frontiers in Number Theory, Physics, and Geometry. II, pp. 3-65. Springer, Berlin (2007)

Publisher's Note Springer Nature remains neutral with regard to jurisdictional claims in published maps and institutional affiliations. 